\title{
Improved heavy quark potential at finite temperature from anti-de Sitter supergravity
}

\author{
Satchidananda Naik * \\ Mehta Research Institute of Mathematics \\ and Mathematical Physics \\ Chhatnag Road, Jhusi \\ Allahabad-211 019, INDIA
}

May 19, 2018

*e-mail: naik@mri.ernet.in 


\begin{abstract}
We improve the heavy quark potential, extracted from the Wilson loop average in the Ads/CFT approach by taking the quantum fluctuation of the only radial coordinate of $A d s_{5}$ which is transverse to the world-sheet of the classical Nambu-Goto string in the static gauge, and obtain the universal Lüscher-Symanzik-Weisz/Lüscher term.
\end{abstract}


A seminal conjecture by Maldacena [1] states the duality between the large $N$ superconformal Yang-Mills theory and the low energy behaviour of the superstring theory in the Anti-de Sitter space back-ground. It is further shown that the correlation functions of the local gauge invariant operators of $\mathcal{N}=4$ supersymmetric Yang-Mills theory are related to the classical limit of the superstring partition function [2, 3]. Also the vacuum expectation values of the non-local operators like Wilson loops are easily computed using this duality [4, 5]. This facilitates to extract the heavy quark potential for SUSY Yang-Mills theories. The proposal became quite useful after Witten [6] has shown how one can break Supersymmetry with non-zero temperature. This makes a close contact with the pure QCD and as far as possible, several qualitative behaviour of QCD in the strong coupling limit are verified [8]. In this approach the background Euclidean time is compactified on a circle which is related to the equilibrium temperature T. Here one takes the space-time fermion to be anti-periodic in this direction and then all the fermions will be as heavy as the order of the temperature $\mathrm{T}$. In the extreme high temperature limit fermions decouple. Thus the $\mathrm{d}+1$ dimensional theory is described as a d dimensional theory without any fermion. This gives us a d dimensional pure QCD in the zero temperature.

The heavy quark potential of the planar $Q C D_{3}$ is extracted from the vacuum expectation value of the Wilson loop operator [5, 7]. The space-like Wilson loop gives a linear potential which correctly signals the area law. However the LüscherSymanzik-Weisz (LSW) term or commonly known as Lüscher term is absent from this potential [9]. This is a universal term independent of any coupling constant and is a characterstic of the string generated QCD potential and has been seen and correctly estimated by Lattice simulation [10]. This might cast doubt on the supergravity approach to study planar QCD. However there are some attempts by Greensite and Olesen [11] and by Dorn and Otto [12] to get rid of this problem. So far there is no concrete explanation in this regard. Here we claim to get this LSW term by considering quantum fluctuations of the classical solution which is an improvement over the Nambu-Goto solution taken by authors of Ref. [5, 7] . We will discuss all the points in the sequel.

Here we consider the case of planar $Q C D_{3}$ which can be trivially extended to higher dimension. Following Maldacena [4] we put heavy quark and anti-quark at $x=L / 2$ and $x=-L / 2$ respectively which are attached by a Nambu-Goto string in an $A d s_{5} \times S_{5}$ black-hole background, where the metric reads

$$
d s^{2}=\alpha^{\prime}\left\{\frac{U^{2}}{R^{2}}\left(\left(1-U_{T}^{4} / U^{4}\right) d t^{2}+\sum_{i} d x_{i}^{2}\right)+\frac{R^{2}}{U^{2}} \frac{d U^{2}}{1-U_{T}^{4} / U^{4}}+R^{2} d \Omega_{5}^{2}\right\},
$$

Where $R^{2}=\sqrt{4 \pi g_{s} N}=\sqrt{4 \pi g^{2}{ }_{Y M} N}$, and $U_{T}=\pi R^{2} T$. Here $g_{s}$ is the string coupling and $N$ is the number of coinciding $D_{3}$ branes which gives $U(N)$ Yang-Mills 
theory and $R$ is the radius of the anti-de Sitter space. The Nambu-Goto string action in this background is

$$
S=\frac{1}{2 \pi \alpha} \int d \tau d \sigma \sqrt{\operatorname{det}\left(G_{M N} \partial_{\mu} x^{M} \partial_{\nu} x^{N}\right)} .
$$

We look for the Wilson loop which traces area in the space-like surface. The boundaries are accordingly $x^{1}= \pm \frac{L}{2}, \quad x^{0}=x^{3}=0$ and the line where $U \rightarrow \infty$ runs parallel to $x^{2}$ axis. In the static gauge the world-sheet cordinates are $\tau=x^{2}, \quad \sigma=x^{1}$ and $\partial_{\tau} x^{M}=\delta_{2}^{M}$ and $\partial_{\sigma} x^{0}=\partial_{\sigma} x^{3}=0$. Thus the action

$$
S=\frac{\mathcal{T}}{2 \pi} \int_{-\frac{L}{2}}^{+\frac{L}{2}} d \sigma \sqrt{\frac{U^{4}}{R^{4}}+U^{\prime 2} \frac{U^{4}}{U^{4}-U_{T}^{4}}} .
$$

The potential is extracted in the $\mathcal{T} \rightarrow \infty$ limit as

$$
V(L)=\operatorname{Lim}_{\mathcal{T} \rightarrow \infty} \frac{1}{\mathcal{T}} S_{c l}
$$

The $\mathcal{T} \rightarrow \infty$ limit is the analouge of $\hbar \rightarrow 0$ limit and thus the classical solution is an exact solution. However Lüscher term is a purely quantum mechanical term showing the finite size scaling. In this very brief report we show here how this classical potential is extracted and how it can be further improved to accommodate the LSW/Lüscher term.

Using symmetry and the classical equation of motion we get

$$
\sqrt{\frac{U^{4}}{R^{4}}+U^{\prime 2} \frac{U^{4}}{U^{4}-U_{T}^{4}}}=C_{0} \frac{U^{4}}{R^{4}}
$$

Where $C_{0}$ is a constant which is fixed from the boundary relation $U\left(\sigma_{0}\right)=U_{0}$ is such that $U^{\prime}\left(\sigma_{0}\right)=0$. This gives

$$
\frac{L}{2}-\sigma=\frac{R^{2}}{U_{0}} \int_{U / U_{0}}^{\infty} \frac{d y}{\sqrt{\left(y^{4}-1\right)\left(y^{4}-1+\epsilon\right)}}
$$

where $\epsilon=1-U_{T}^{4} / U_{0}^{4}$ and the dimension less variable $y(\sigma)=\frac{U(\sigma)}{U_{0}}$. The classical action is

$$
S=\frac{\mathcal{T}}{2 \pi} U_{0} \int_{1}^{\infty} d y \frac{y^{4}}{\sqrt{\left(y^{4}-1\right)\left(y^{4}-1+\epsilon\right)}},
$$

which gives

$$
V(L)=\frac{U_{0}^{2}}{2 \pi R^{2}} L+\frac{U_{0}}{\pi} \int_{1}^{\infty} d y\left(\sqrt{\frac{y^{4}-1}{y^{4}-1+\epsilon}}-1\right)+\frac{U_{0}-U_{T}}{\pi} .
$$


This is a linear potential $V(L)=\sigma L+\cdots$ where $\sigma=\frac{U_{0}^{2}}{2 \pi R^{2}}$ is the string tension. In terms of Yang-Mills coupling this gives $\sigma=\frac{\pi}{\sqrt{2}} g_{Y M} \sqrt{N T^{3}}$. However the subleading term $\frac{c}{L}$ is missing in the classical approximation. Also $c$ is supposed to be universal term which does not contain any physical parameter except the finite size scale $L$. This is a purely quantum effect. We take here one-loop quantum effect around the classical solution of $U$ as $U=U_{c l}+\eta$ where $\eta$ is the quantum field. The action in this order is given by

$$
S_{2}=\frac{\mathcal{T}}{2 \pi} \int_{\sigma_{0}}^{L / 2} \mathcal{L}_{2}
$$

where

$$
\mathcal{L}_{2}=\frac{\partial^{2} \mathcal{L}}{\partial U^{2}} \eta^{2}+2 \frac{\partial^{2} \mathcal{L}}{\partial U \partial U^{\prime}} \eta \partial \eta+\frac{\partial^{2} \mathcal{L}}{\partial U^{\prime 2}}(\partial \eta)^{2}
$$

Here $\mathcal{L}$ is the Lagrangian given in eq.(3). To integrate over the field $\eta$ we redefine

$$
\varphi=\alpha \eta
$$

where $\alpha$ is a function of the classical field $U_{c l}$ obeying equations of motion. (Henceforth we denote $U$ as $U_{c l}$ and drop the subscript.) Now $\eta$ is substituted as $\frac{\varphi}{\alpha}$ in eq.(10) to give

$$
\mathcal{L}_{2}=(\partial \varphi)^{2}-2 \varphi \partial \varphi\left(\frac{\alpha^{\prime}}{\alpha}-\frac{b}{2 \alpha^{2}}\right)+\left(\frac{\alpha^{\prime 2}}{\alpha^{2}}-b \frac{\alpha^{\prime}}{\alpha^{3}}+\frac{c}{\alpha^{2}}\right) \varphi^{2}+\mathcal{L}_{\text {measure }}
$$

and $\mathcal{L}_{\text {measure }}$ is due to the redefinition of the field $\eta$, where

$$
\begin{aligned}
\alpha^{2} & =\frac{\partial^{2} \mathcal{L}}{\partial U^{\prime 2}} \\
b & =2 \frac{\partial^{2} \mathcal{L}}{\partial U \partial U^{\prime}} \\
c & =\frac{\partial^{2} \mathcal{L}}{\partial U^{2}} .
\end{aligned}
$$

( Here $/$ denotes the derivative with respect to $\sigma$.) Any generic term like $f \partial \varphi \varphi$ can be taken as $\left.\frac{1}{2} f \varphi \varphi\right|_{\text {bounadry }}$ and a term like $-\frac{1}{2} \partial f \varphi \varphi$ due to integration by parts in the action. We take here the Dirichlet boundary condition for the fluctuating field $\varphi$ and hence drop the boundary term from the action. This gives

$$
\mathcal{L}_{2}=\left((\partial \varphi)^{2}+\left(\frac{\alpha^{\prime \prime}}{\alpha}-\frac{1}{2} \frac{b^{\prime}}{\alpha^{2}}+\frac{c}{\alpha^{2}}\right) \varphi^{2}\right)+\mathcal{L}_{\text {measure }}
$$

Taking further derivatives with respect to $\sigma$ of the equation of motion

$$
\partial \frac{\partial \mathcal{L}}{\partial U^{\prime}}=\frac{\partial \mathcal{L}}{\partial U}
$$


one gets

$$
\frac{c}{\alpha^{2}}-\frac{1}{2} \frac{b^{\prime}}{\alpha^{2}}=2 \frac{U^{\prime \prime}}{U^{\prime}} \frac{\alpha^{\prime}}{\alpha}+\frac{U^{\prime \prime \prime}}{U^{\prime}} .
$$

Substituting this we get

$$
\mathcal{L}_{2}=(\partial \varphi)^{2}+m^{2}(\sigma) \varphi^{2}
$$

where the effective mass like term

$$
m^{2}(\sigma)=\left(\partial^{2} \alpha+2 \frac{U^{\prime \prime}}{U^{\prime}} \partial \alpha+\frac{U^{\prime \prime \prime}}{U^{\prime}} \alpha\right) \frac{1}{\alpha} .
$$

From eq.(13) $\alpha$ is given by

$$
\alpha=\frac{g(\sigma)}{U^{\prime}(\sigma)}
$$

where

$$
g(\sigma)=\frac{U_{0}}{R} \frac{\sqrt{y^{4}-1}}{y^{2}} .
$$

This shows that

$$
m^{2}(\sigma)=\frac{\partial^{2} g}{g} .
$$

From eq.(6) one observes that $y(\sigma)$ remains infinitesimally closer to 1 and then rapidly grows to infinity. If we take $y(\sigma)=1+\delta(\sigma)$ where $\delta$ is a very small parameter and keeping up to first order we get

$$
\frac{L}{2}-\sigma=\text { const. } \sqrt{\delta(\sigma)}
$$

and for $y(\sigma)$ little bigger we can neglect 1 compare to $y^{4}$ in eq.(6) and get

$$
\frac{L}{2}-\sigma=\text { const. } \frac{1}{y^{3}} .
$$

For the first case (c.f. eq.(21) and eq.(23))

$$
g(\sigma)=p(1-\sigma)
$$

where $p=2 U_{0}^{3} \frac{\sqrt{\epsilon}}{R^{3}}$ which gives $\partial^{2} g=0$ and for $y$ large $g(\sigma)$ is a constant and $\partial^{2} g=0$. This shows that $m^{2}(\sigma)$ is zero for the entire range of integration.

The measure is now changed from $D \eta \rightarrow D \varphi$ and the contribution of this to the effective action

$$
S_{\text {measure }}=\frac{1}{L} \int_{\sigma_{0}}^{L / 2} d \sigma \ln \alpha^{2},
$$


This integration can be trivially done which contributes a constant to the potential. We take the quantum fluctuation $\eta$ to be zero on the boundary of the Wilson loop i.e. for $\sigma=0$ and $L / 2$. Then the integration over $\varphi$ gives the most required LSW term $-\frac{\pi}{12 L}$ for the potential which is our main result.

As mentioned earlier the classical action is exact in the static gauge when $\mathcal{T} \rightarrow$ $\infty$. We just supplemented this classical action with the quantum fluctuation of the transverse cordinate $U$. We do not take the fluctuations of the world-sheet for all the transverse coordinate as has been shown by Greensite and Olesen [13]. In the finite temperature case and the high temperature limit when dimensional reduction is taking place, fermions completely decouple from the spectrum. Since we are in the very low energy limit of extracting the potential we feel it is legitimate not to take any of these fluctuations.

\section{Note Added}

This work was completed in December and was presented in the String theory workshop at Puri (9th Dec- 19th Dec 1998). That time I was not aware of the work of Greensite and Olesen [13] and also of Förste et. al. [14] where both the groups have considered the worldsheet fluctuations of both the fermionic and the bosonic coordinates.

Acknowledgements: I would like to thank the organisers and all the participants of the Puri workshop for fruitful discussions. 


\section{References}

[1] J. Maldacena, Adv. Theor. Math. Phys. 2 (1998) 231,

[2] S.S. Gubser, I. Klebanov, A.M.Polyakov Phys. Lett. B 428 (1998)105

[3] E. Witten, Adv. Theor. Math. Phys.2 (1998) 253,

[4] J. Maldacena, Phys. Rev. Lett.80 (1998) 4859,

[5] S-J. Rey and J-T. Yee, hep-th/9803001;

S-J. Rey, S. Theisen, and J-T. Yee, Nucl. Phys.B 527 (1998) 171

[6] E. Witten, Adv. Theor. Math. Phys.2 (1998) 505,

[7] A. Brandhuber, N. Itzhaki, J. Sonnenschein, and S. Yankielowicz, J. High Energy Phys. 06 (1998) 001; Phys. Lett.B 434 (1998) 36,

[8] D. J. Gross and H. Ooguri, Phys. Rev.D 58 (1998) 106002,

C. Csaki, H. Ooguri, Y. Oz, and J. Terning, hep-th/9806021

R. de Mello Koch et al. Phys. Rev D 58 (1998) 105009.

[9] M. Lüscher, K. Symanzik, and P. Weisz, Nucl. Phys.B173 (1980) 365

M. Lüscher, Nucl. Phys.B190 [FS2] (1981) 31.

[10] G. Bali, C. Schlichter, and K. Schilling, Phys. Rev.D51 (1995) 5165.

[11] J. Greensite and P. Olesen, J. High Energy Phys 08 (1998) 009,

[12] H. Dorn, H.J. Otto , J. High Energy Phys 09 (1998) 021.

[13] J. Greensite and P. Olesen, Worldsheet fluctuations and heavy quark potential in the Ads/CFT approach hep-th-9901057

[14] R.Kallosh and A.A.Tseytlin , J. High Energy Phys 10 (1998) 016 S. Förste, D. Ghoshal, S.Theisen, Stringy corrections to Wilson loop in $N=4$ Super Yang-Mills theory hep-th- 9903042 